\title{
La Deiras revisitée
}

Nouvelles études sur la nécropole mycénienne d'Argos

Anna Philippa-Touchais, Gilles Touchais, Nikolas Papadimitriou, Evangelia Pappi, Niki Papakonstantinou et Sevi Triantaphyllou

\section{OpenEdition}

12 Journals

Édition électronique

URL : http://journals.openedition.org/baefe/1720

DOI : 10.4000/baefe. 1720

ISSN : 2732-687X

Éditeur

ResEFE

Référence électronique

Anna Philippa-Touchais, Gilles Touchais, Nikolas Papadimitriou, Evangelia Pappi, Niki

Papakonstantinou et Sevi Triantaphyllou, « La Deiras revisitée » [notice archéologique], Bulletin archéologique des Écoles françaises à l'étranger [En ligne], Grèce, mis en ligne le 26 novembre 2020, consulté le 22 mars 2021. URL : http://journals.openedition.org/baefe/1720 ; DOI : https://doi.org/ $10.4000 /$ baefe. 1720

Ce document a été généré automatiquement le 22 mars 2021

Le Bulletin archéologique des Écoles françaises à l'étranger est mise à disposition selon les termes de la Licence Creative Commons Attribution - Pas d'Utilisation Commerciale - Pas de Modification 4.0 International. 


\section{La Deiras revisitée}

Nouvelles études sur la nécropole mycénienne d'Argos

Anna Philippa-Touchais, Gilles Touchais, Nikolas Papadimitriou, Evangelia Pappi, Niki Papakonstantinou et Sevi Triantaphyllou

\section{NOTE DE L'AUTEUR}

Autorité nationale présente : Evangelia Pappi (Éphorie des antiquités d'Argolide)

Numéro de mission : B07

Composition de l'équipe : Yannis Bassiakos (Centre Demokritos) et Myrto Georgakopoulou (BSA) : analyses des objets en bronze et en or ; Harikleia Brécoulaki (Ethniko Idryma Erevnon/KERA) et Sofia Sotiropoulou (Ormylia Art Diagnosis Center) : analyses des peintures murales ; Cydrisse Cateloy (Université Paris 1) : étude des amphores cananéennes; Éléni Drakaki (Ministère de la Culture) : étude des sceaux ; Akis Goumas (orfèvre, technologue) : étude technologique des ors et des ivoires ; Maria Kayafa (Ministère de l'Éducation) : étude des objets en bronze ; Vassilis Kilikoglou (Centre Demokritos) et Kelly Christodoulou (Université d'Athènes) : analyses et étude des vases recouverts d'étain ; Éléni Konstantinidi-Syvridi (Musée national d'Athènes) et Anna Philippa-Touchais (EFA-UMR 7041) : étude des bijoux en or ; Nikos Papadimitriou (Université de Heidelberg) : étude architecturale des tombes ; Evangelia Pappi (Éphorie des antiquités d'Argolide) : étude de la céramique géométrique ; Kostas Paschalidis (Musée national d'Athènes) : étude de la céramique mycénienne ; Malgorzata Siennicka-Rahmstorf (Université de Copenhague) : étude des empreintes de textile ; Mélina Smyrniou (University College, London) : étude des perles en verre et faïence ; Gilles Touchais (Université Paris 1 - UMR 7041) : histoire des fouilles, travail éditorial ; Iphiyénia Tournavitou (Université de Thessalie) : étude des ivoires ; Sevi Triantaphyllou et Niki Papakonstantinou (Université de Thessalonique) : étude des ossements humains. Partenariats institutionnels : Musée national d'Athènes, Éphorie des antiquités d'Argolide Établissement porteurs de l'opération : École française d'Athènes, Université Paris 1, UMR 7041 « ArScAn » 
Données scientifiques produites :

Argos par l'EFA

$1 \mathrm{Au}$ cours des années 2016 à 2018, le programme d'étude du mobilier des tombes mycéniennes de la Deiras fouillées jadis par Vollgraff a été poursuivi. L'effort a porté, d'une part, sur la céramique géométrique ${ }^{1}$, dont l'étude a été poursuivie au Musée archéologique d'Argos et au Musée national d'Athènes; d'autre part, sur le matériel anthropologique issu des tombes I et VIII. Parallèlement, on a commencé de préparer le manuscrit du volume dans lequel seront présentés les résultats de l'ensemble des nouvelles recherches menées depuis 2010 sur la nécropole de la Deiras, volume à paraître dans la série des Études Péloponnésiennes sous le titre La Deiras revisitée.

\section{La céramique géométrique}

2 Au Musée archéologique d'Argos, l'examen de la céramique géométrique provenant des fouilles du dromos de la tombe $\mathrm{V}$ a été poursuivie par E. Pappi, responsable de ce dossier. Une centaine de fragments, retenus pour la publication, ont été catalogués, documentés et dessinés.

$3 \mathrm{Au}$ Musée national d'Athènes (MNA), un lot de céramique géométrique portant l'indication «ARGOS ? Fouille Vollgraff » avait été repéré il y a trois ans dans la réserve de la Collection préhistorique, lors de l'étude de la céramique mésohelladique des premières fouilles de l'Aspis ${ }^{2}$. Supposant que ce matériel provenait de la nécropole mycénienne de la Deiras et souhaitant donc l'inclure dans le volume en préparation, nous l'avons examiné : il s'agit d'un lot de 500 tessons environ, datant pour l'essentiel du Géométrique Récent et portant pour plusieurs d'entre eux un décor figuré.

4 Au cours du travail d'inventaire, E. Pappi a constaté qu'un certain nombre de tessons appartenaient en fait au matériel géométrique découvert par Vollgraff lors des fouilles de la Larissa (1928-1930) et publié par A. Roes en $1953^{3}$ : une trentaine de tessons qui sont illustrés sur les figures et les planches de l'article de Roes (fig. 2, 4, 6 et 8) ont en effet pu être reconnus (fig. 1, 3, 5 et 7). Ce lot de céramique ne provient donc pas de la nécropole de la Deiras mais de l'acropole de la Larissa. 
Fig. 1. Tessons géométriques du MNA identifiés avec des tessons publiés provenant de la Larissa.

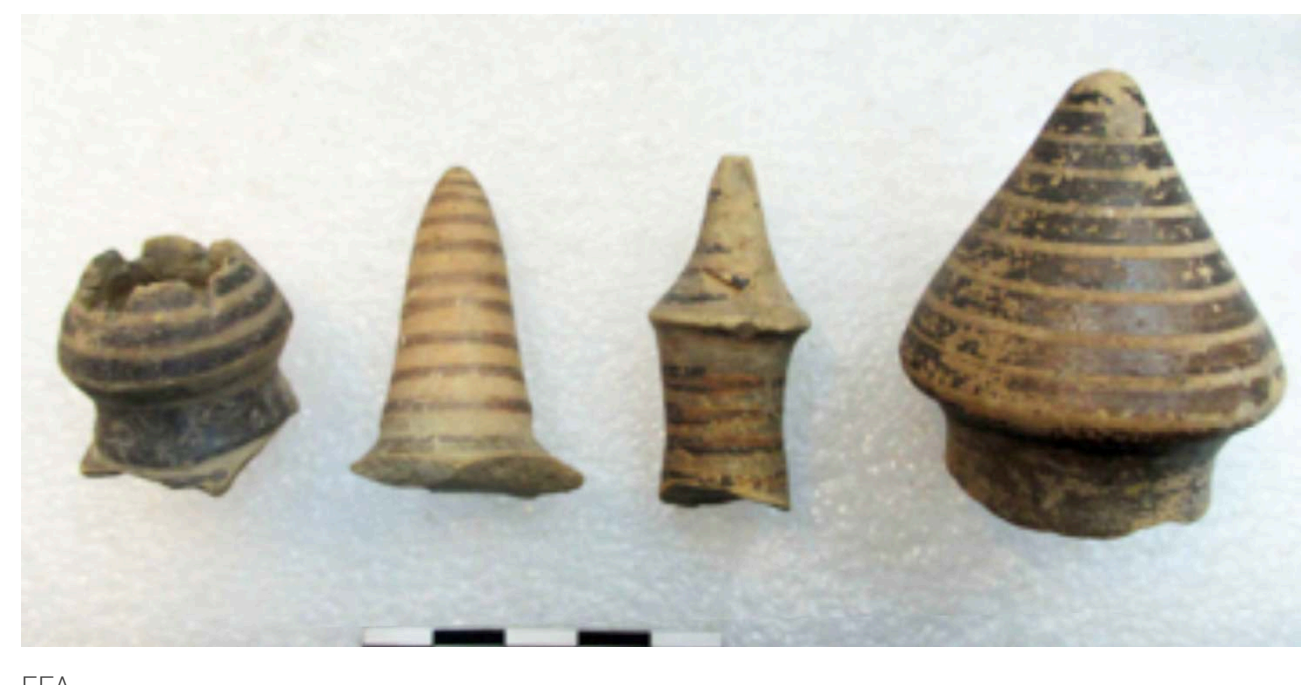

Fig. 2. Tessons géométriques du MNA identifiés avec des tessons publiés provenant de la Larissa.
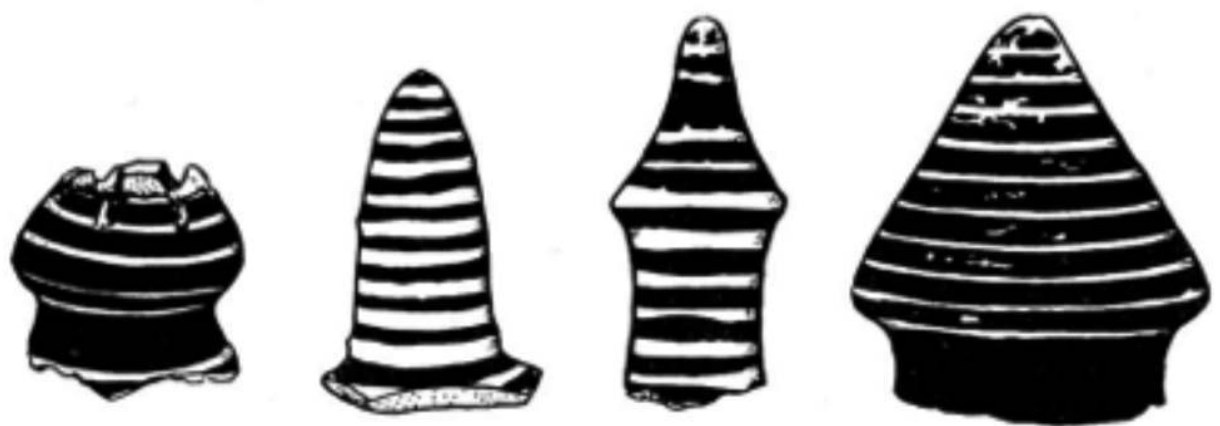

Fig. 7. - Beutons de couvercles de pyxis, Larissa.

EFA/ROES 1953, p. 101, fig. 7. 
Fig. 3. Tesson géométrique du MNA identifié avec un tesson publié provenant de la Larissa.

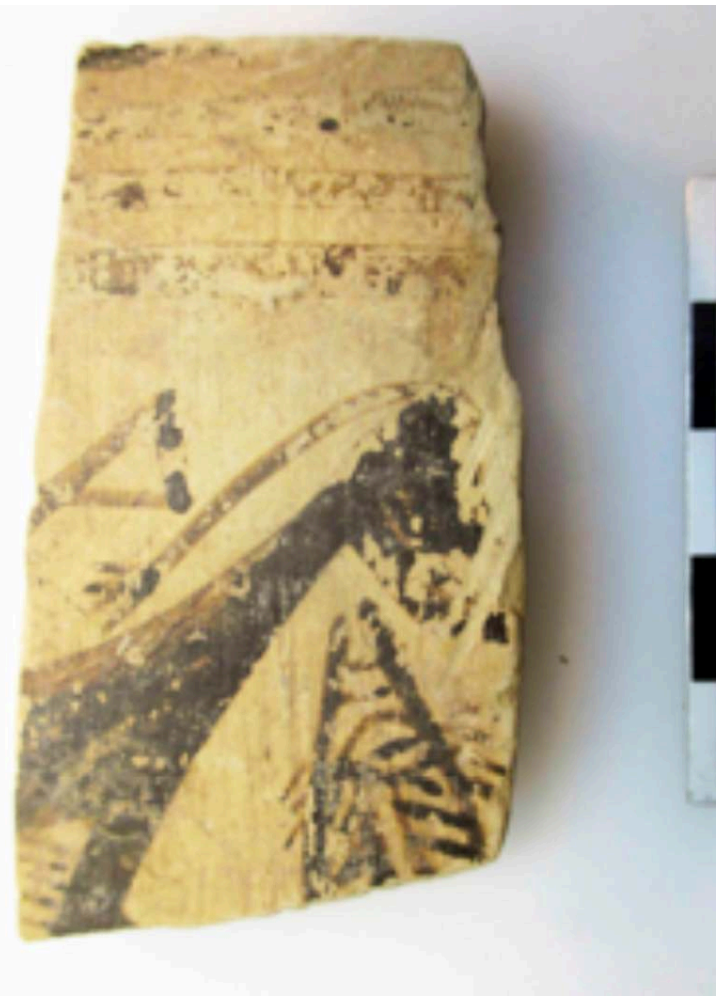

EFA.

Fig. 4. Tessons géométriques du MNA identifiés avec des tessons publiés provenant de la Larissa.

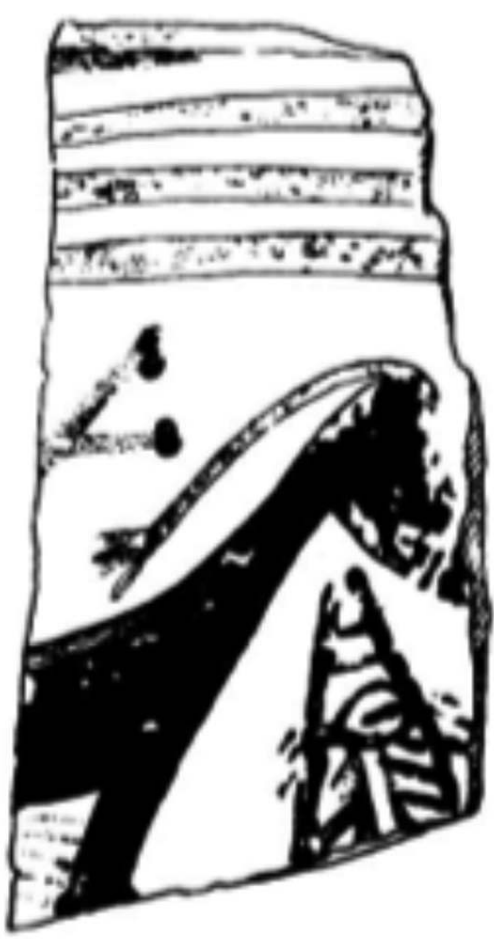

EFA/ROES 1953, p. 98, fig. 4. 
Fig. 5. Tessons géométriques du MNA identifiés avec des tessons publiés provenant de la Larissa.

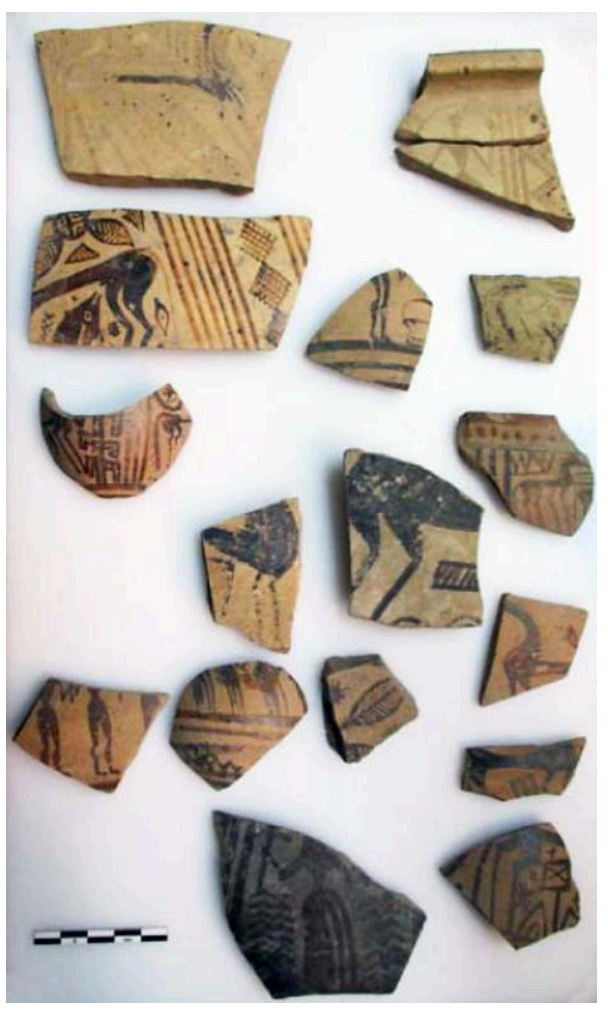

EFA.

Fig. 6. Tessons géométriques du MNA identifiés avec des tessons publiés provenant de la Larissa.

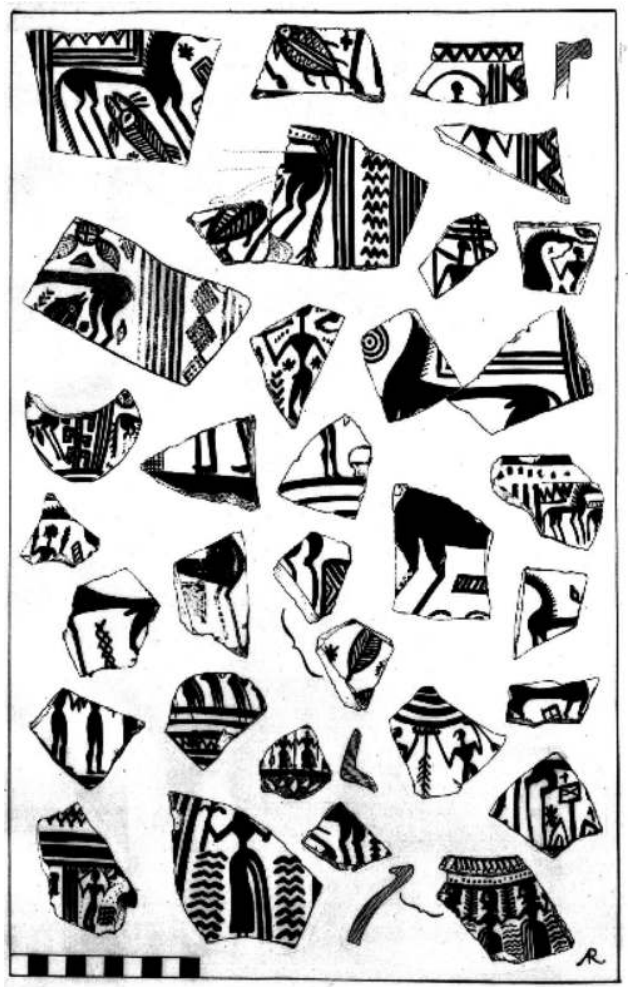

EFA/Roes 1953, pl. XXIV.

Bulletin archéologique des Écoles françaises à l'étranger , Grèce 
Fig. 7. Tessons géométriques du MNA identifiés avec des tessons publiés provenant de la Larissa.

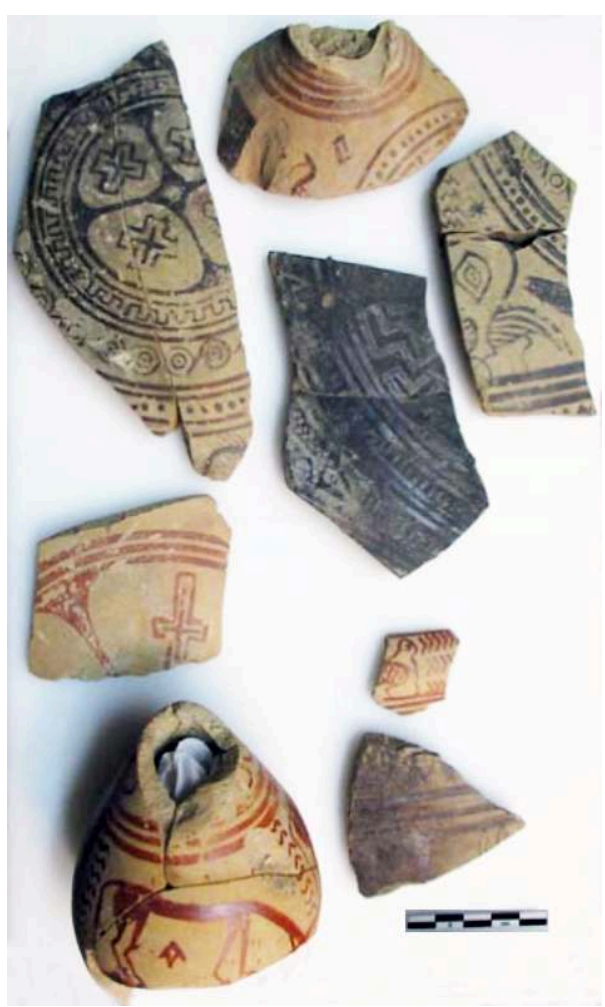

EFA.

Fig. 8. Tessons géométriques du MNA identifiés avec des tessons publiés provenant de la Larissa.

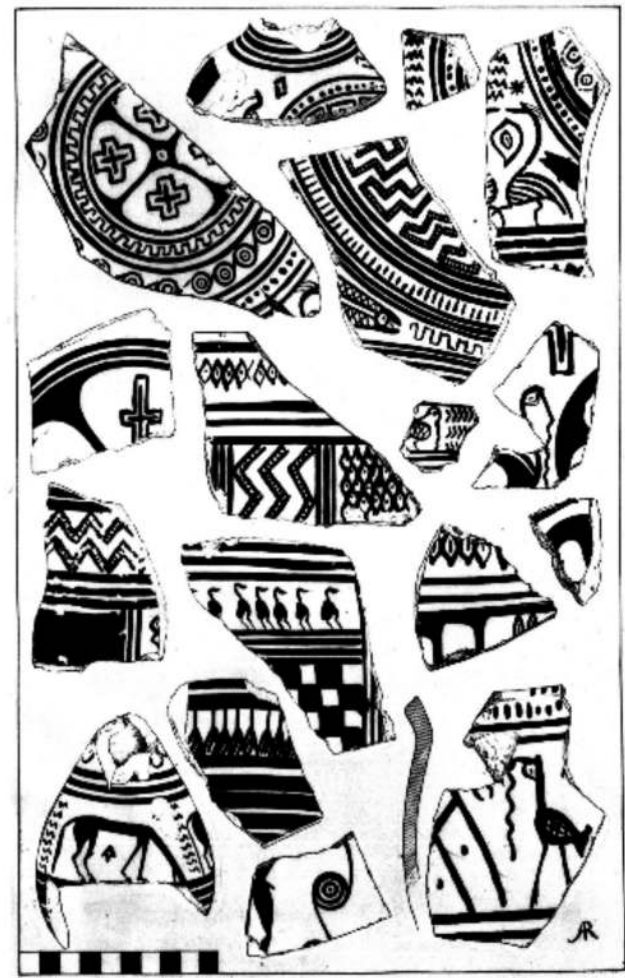

EFA/Roes 1953, pl. XXV.

Bulletin archéologique des Écoles françaises à l'étranger , Grèce 
Cette trouvaille est néanmoins intéressante à plusieurs titres. D'abord parce qu'un ensemble céramique du Musée national dont la provenance était incertaine est désormais clairement identifié. Ensuite, parce que le matériel - que l'on croyait en grande partie perdu - des fouilles conduites par Vollgraff dans un sanctuaire géométrico-archaïque situé au sommet de la Larissa a finalement été localisé. Enfin, parce que l'étude détaillée et la publication de l'ensemble de ce matériel - dont 150 tessons seulement étaient connus -, enrichiront à coup sûr notre connaissance de l'un des plus anciens sanctuaires d'Argos, surtout si on les mène en liaison avec celles des nombreux objets inédits du musée d'Argos issus des fouilles de Vollgraff et de Courbin dans le même secteur ${ }^{4}$.

6 C'est pourquoi l'on envisage de poursuivre l'étude de cet ensemble céramique du Musée national d'Athènes dans le cadre d'un volume collectif, édité par l'École française d'Athènes, qui présenterait les diverses séries de trouvailles (Vollgraff, Courbin) liées à l'implantation des premiers sanctuaires sur la Larissa aux époques géométrique et archaïque. Ce projet, qui s'accompagnera d'un relevé précis des vestiges architecturaux, a d'ores et déjà reçu l'aval des autorités.

\section{Le matériel anthropologique}

7 L'étude des seuls ossements recueillis par Vollgraff qui nous sont parvenus a été réalisée par N. Papakonstantinou en collaboration avec S. Triantaphyllou. Pour la tombe I, l'examen approfondi des fragments osseux partiellement calcinés ${ }^{5}$ visait à déterminer la nature exacte de l'assemblage : sépulture à incinération (hypothèse du fouilleur) ou restes de fumigation ? L'analyse des 478 fragments, ainsi que les nombreux recollages effectués (fig. 9), ont permis de déterminer la présence d'au moins sept individus ( 5 adultes des deux sexes et 2 subadultes) et d'écarter l'hypothèse de la crémation au profit de celle de la fumigation, pratique beaucoup plus ordinaire dans les tombes à chambre mycéniennes.

8 L'examen des quelque 29 fragments d'os post-crâniens identifiables provenant de la tombe VIII a confirmé le NMI de deux individus adultes ${ }^{6}$ et permis d'observer, sur un fragment, des stigmates de périostitite. 
Fig. 9. L'assemblage osseux de la tombe I après tri et recollages.

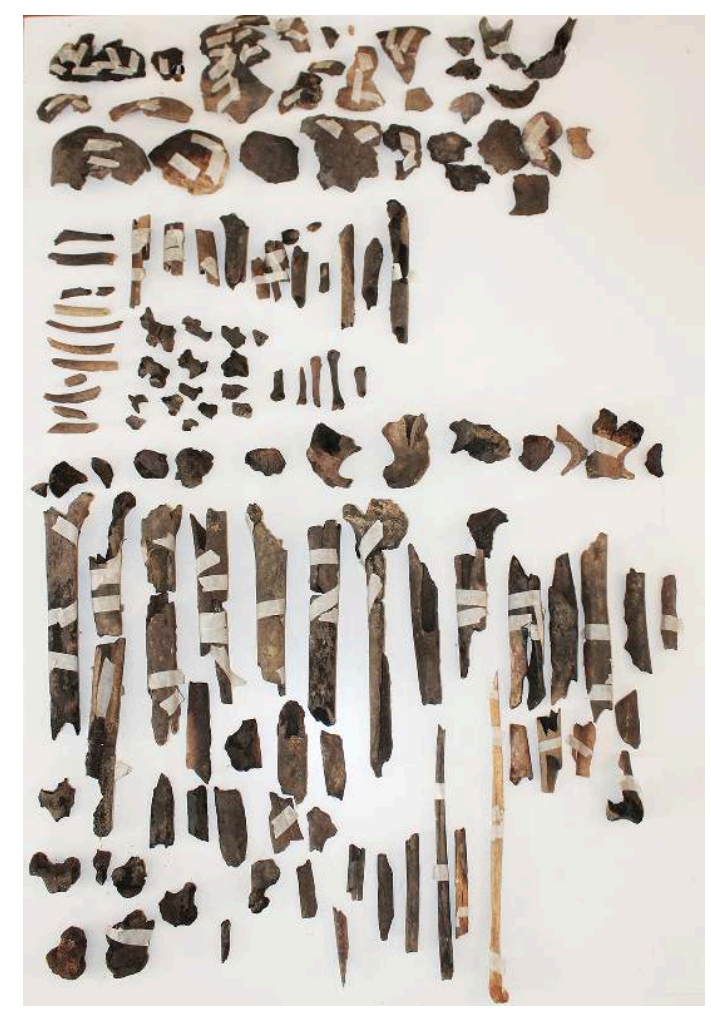

EFA.

\section{Bibliographie}

Yannis Bassiakos, Georgios Mastrotheodoros, Maria Kayafa, Nikolas Papadimitriou,

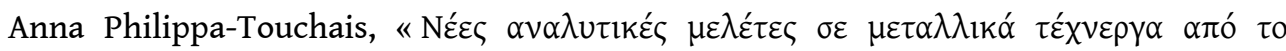

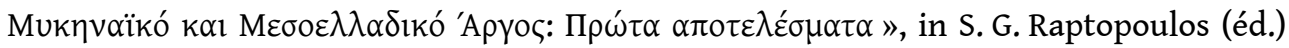

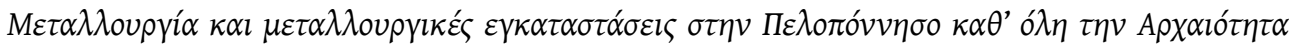

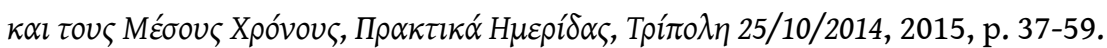

Jean Deshayes, Argos. Les fouilles de la Deiras, Athènes, EFA, Ét. Pélop IV, 1966.

Eleni Konstantinidi-Syvridi, Nikolas Papadimitriou, Anna Philippa-Touchais, Akis Goumas, « Goldworking techniques in Mycenaean Greece (17th/16th-12th century BC): some new observations ", in Harald Meller, Roberto Risch, Ernst Pernicka (éd.), Metalle der Macht- Frühes Gold und Silber, Halle, Landesmuseum für Vorgeschichte, Tagungen des Landesmuseums für Vorgeschichte Halle 11/I, 2014, p. 335-348.

Nikolas Papadimitriou, Anna Philippa-Touchais, Gilles Touchais, "The Mycenaean cemetery of Deiras in a local and regional context », in Joanne M. A. Murphy (éd.), Death in Late Bronze Age Greece. Variations on a Theme, Oxford, OUP, 2020, p. 60-88.

Anna Philippa-Touchais, Nikolas Papadimitriou, «Deiras, Argos: The Mycenaean cemetery revisited in the light of unpublished finds from W. Vollgraff's excavations ", in Ann-Louise Schallin, Iphiyenia Tournavitou (éd.), Mycenaeans up to Date: The Archaeology of North-Eastern Peloponnese. Current Concepts and New Directions, Stockholm, Swedish Institute at Athens, ActaAth, 56, 2015, p. 449-467. 
14 Anna Philippa-Touchais, Nikolas Papadimitriou, Gilles Touchais, «Travaux de l'École française d'Athènes, II. Rapports 2011. Argos. La Deiras ; III. Rapports 2012. Argos. La Deiras », BCH 136-137 (2012-2013), 2015, p. 612-621 et 817-824. Anna Philippa-Touchais, Nikolas Papadimitriou, Gilles Touchais, «Rapports sur les travaux de l'École française d'Athènes en 2013. Argos. La Deiras », BCH 138, 2014, p. 749-756. Anna Philippa-Touchais, Nikolas Papadimitriou, Gilles Touchais, «Rapports sur les travaux de l'École française d'Athènes en 2014 et en 2015. Argos. La Deiras ", BCH 139-140, 2015-2016, 2018, p. 828-842. Anne Roes, "Fragments de poterie géométrique trouvés sur les citadelles d'Argos ", BCH 77, 1953, p. 90-104. Wilhelm Vollgraff, «Fouilles d'Argos. A. - La nécropole mycénienne de la Deiras », BCH 28,1904, p. 364-399.

\section{BIBLIOGRAPHIE}

ROES 1953

Anne Roes, « Fragments de poterie géométrique trouvés sur les citadelles d'Argos », $B C H$ 77, 1953, p. 90-104.

BCH 54, 1930

«Chronique des fouilles et découvertes archéologiques dans l'Orient hellénique », BCH 54, 1930.

BCH 79, 1955

«Chronique des fouilles et découvertes archéologiques en Grèce en 1954 », BCH 79, 1955.

BCH 136-137, 2012-2013

Anna Philippa-Touchais, Nikolas Papadimitriou, Gilles Touchais, « Travaux de l'École française d'Athènes, II. Rapports 2011. Argos. La Deiras ; III. Rapports 2012. Argos. La Deiras », BCH 136-137 (2012-2013), 2015, p. 612-621 et 817-824.

BCH 138, 2014

Anna Philippa-Touchais, Nikolas Papadimitriou, Gilles Touchais, « Rapports sur les travaux de l'École française d'Athènes en 2013. Argos. La Deiras », BCH 138, 2014, p. 749-756.

BCH 139-140, 2015-2016

Anna Philippa-Touchais, Nikolas Papadimitriou, Gilles Touchais, « Rapports sur les travaux de l'École française d'Athènes en 2014 et en 2015. Argos. La Deiras ", BCH 139-140, 2015-2016, 2018, p. 828-842.

\section{NOTES}

1. $B C H$ 139-140, 2015-2016, p. 840-842.

2. $B C H 136-137,2012-2013$, p. 808-809. 
3. ROES 1953, p. 90-104.

4. $B C H 54,1930$, p. 480 ; BCH 79, 1955, p. 314.

5. BCH 136-137, 2012-2013, p. 617-618.

6. BCH 136-137, 2012-2013, p. 619.

INDEX

Thèmes : EFA

chronologie https://ark.frantiq.fr/ark:/26678/pcrtxT02uJOogm, https://ark.frantiq.fr/ark:/ 26678/pcrtGTWPtWn8qu

lieux https://ark.frantiq.fr/ark:/26678/pcrtRm0r24dwUy

Année de l'opération : 2016, 2017, 2018

sujets https://ark.frantiq.fr/ark:/26678/pcrtsIm3RuNMGu, https://ark.frantiq.fr/ark:/26678/ pcrtMw5xXEAy1F, https://ark.frantiq.fr/ark:/26678/pcrt1DMOWvDF4j, https://ark.frantiq.fr/ ark:/26678/crtiMNlD8Asde

\section{AUTEURS}

ANNA PHILIPPA-TOUCHAIS

UMR 7041 «ArScAn »

\section{GILLES TOUCHAIS}

Université Paris 1, UMR 7041 « ArScAn »

\section{NIKOLAS PAPADIMITRIOU}

Université de Heidelberg

\section{EVANGELIA PAPPI}

Éphorie des antiquités d'Argolide

\section{NIKI PAPAKONSTANTINOU}

Université de Thessalonique

\section{SEVI TRIANTAPHYLLOU}

Université de Thessalonique 